\title{
Hair Follicle miRNAs: a Novel Biomarker for Primary Blast Induced-Mild Traumatic Brain Injury
}

Jing Zhang ${ }^{1,4}$, Rosalinda Knight ${ }^{2}$, Yushan Wang ${ }^{3}$, Thomas W. Sawyer ${ }^{3}$, Christopher J. Martyniuk $^{2,6}$ and Valerie S. Langlois ${ }^{1,5^{*}}$

${ }^{1}$ Chemistry and Chemical Engineering Department, Royal Military College of Canada, Kingston, ON, Canada, K7K 7B4

${ }^{2}$ University of New Brunswick and Canadian River Institute, NB, Canada, E2L 4L5

${ }^{3}$ Defence Research and Development Canada - Suffield Research Centre, Box 4000, Medicine Hat, AB, Canada, T0J 2N0

\section{Present:}

${ }^{4}$ Schulich School of Medicine \& Dentistry, Western University, ON, N6A 5C1, Canada

${ }^{5}$ Institut national de la recherche scientifique Centre Eau Terre Environnement (INRS-ETE)

490, rue de la Couronne, Québec (Québec), Canada G1K 9A9

${ }^{6}$ University of Florida, Center for Environmental and Human Toxicology \& Department of

Physiological Sciences, Gainesville, FL, USA 32611

*Author for correspondence and reprint requests:

Valérie Langlois, Ph. D.

Professeure agrégée/Associate Professor

Institut national de la recherche scientifique

Centre Eau Terre Environnement (INRS-ETE)

490, rue de la Couronne, Québec (Québec), Canada G1K 9A9

T: +1.418.654.2547 W: http://www.ete.inrs.ca/valerie-langlois

E: valerie.langlois@inrs.ca 


\begin{abstract}
Context

Due to the wide use of improvised explosive devices during modern warfare, primary blastderived mild traumatic brain injury (mTBI) has become a major medical condition in the military. With minimal visually identifiable symptoms, an effective molecular biomarker system is desirable.

Objective

We assessed the potential of mammalian hair follicle miRNAs as an mTBI biomarker.

Materials and methods

Due to their well-established roles in mTBI molecular pathology, the expression level of miR183, miR-26a, miR-181c, miR-29a, miR-34a, and miR-27b was determined using qRT-PCR in whisker hair follicles from rats subject to head-only exposure to a single pulse shock wave. Based on established transcriptomics profiles, sub-network enrichment analysis (SNEA) was also conducted.

\section{Results}

The results reveled that molecular networks involving miR-183, miR-26a, and miR-181c were enriched in multiple treatments whereas sub-networks of miR-29a, miR-34a, and miR-27b were unique to individual exposure groups.
\end{abstract}

\title{
Discussion
}

Our study showed that all six miRNAs were reflective of the mTBI signature involved in cellular responses. Noteworthy was that the decrease in the transcript levels of miR-181c was correlated to shockwave exposure severity.

\section{Conclusion}

This study demonstrates for the first time that mammalian hair follicles are capable of housing miRNA biomarkers for TBI.

Keywords: mTBI, sub-network enrichment analysis, gene set enrichment analysis, transcriptomics, head trauma 


\section{Introduction}

The wide adoption of improvised explosive devices (IEDs) is considered to be the main cause for blast-induced traumatic brain injury (TBI; Ling et al., 2009; Nakagawa et al., 2011). Thanks to the advancements in body armour design and modern operational medicine over the years, the head injury introduced by the penetrating projectiles and impact forces during the initial explosion can be greatly reduced. Additionally, these types of head injuries are less likely to occur at a "far-field" distance. However, the head exposure to the pressure wave generated during explosion can still lead to brain injuries, which are largely composed of cellular/subcellular damages (Jordan, 2013; Sawyer et al., 2016). In such cases, the TBI derived from primary blast is closely associated to mild TBI (mTBI). Indeed, mTBI is responsible for the majority of TBI cases in US military (Laurer and McIntosh, 1999; Bramlett and Dietrich, 2004; Defense and Veterans Brain Injury Center, 2013). Because visually identifiable symptoms are rarely present for mTBI, the diagnosis and effective implement of proper precautions are often not timely. As such, an effective molecular biomarker system that is both effective and reflective of mTBI is needed. As demonstrated previously, the mammalian hair follicle is one such robust system for biomarker assessments (Zhang et al., 2014, 2017, 2018).

MicroRNAs (or miRNA) belong to a 22 nucleotides long small RNA species mostly recognized as a gene expression regulator on a post-transcription and level (He and Hannon, 2004). These small RNAs interact with the seed region of their target mRNA, usually leading to inhibition on translation (Chen and Rajewsky, 2007). MiRNAs have been proposed as biomarkers and/or therapeutic targets for medical disorders (Wang, 2009; Adachi et al., 2010; Mitchell et al., 2008; Hanke et al., 2010). The human genome contains up to 1\% as miRNAs, which target at least 30\% of protein gene transcripts, thereby regulating a range of cellular events 
(Perron et al., 2008; Friedman et al., 2009; Ha and Kim, 2014). In addition, miRNAs are considered to be more stable than other RNA species due to the protective effects from the interaction with lipid vesicles as well as RNA-binding proteins, thus miRNA biomarkers that can be highly stable and informative (Weickmann et al., 1982; Valadi et al., 2007; Gibbings et al., 2009). It is also worth noting that a considerable amount of miRNAs is highly conserved across higher metazoan species (Lee et al., 2007), and it is possible to translate the knowledge obtained from animal models to that of humans. Recent studies have started identifying their roles in TBI responses (Balakathiresan et al., 2012; Sajja et al., 2018). Furthermore, miRNAs have been demonstrated to play critical roles in hair follicle development and functions (Ahmed et al., 2014; Gao et al., 2017; Hochfeld et al., 2017).

Due to their involvement in mTBI molecular pathology, this study explores the potential of hair follicle miRNAs (miR-183, miR-181c, miR-26a, miR-34a, and miR-29a) as biomarkers for primary blast-derived mTBI. It is proposed that glutamate release, calcium overload, mitochondrial dysfunction, axonal responses, neurotransmission alteration, and neuroinflammatory responses are the signature mTBI responses (Walker and Tesco, 2013). Firstly, miR-183 plays key roles in regulating voltage-gated glutamate receptors and synaptic plasticity (Krol et al., 2010; Rizo et al., 2012), and has been proposed as a mTBI marker (Valiyaveettil et al., 2013). It is hypothesized that, in addition to synaptic plasticity, miR-26a exhibits neuro-protective effects through inhibiting cellular calcium overload and mitochondriadependent apoptosis (Zhang et al., 2015; Gu et al., 2015). The miR-181has been shown to be involved in regulating mitochondria genome integrity (Das et al., 2014). Moreover, miR-29a is reportedly part of the inflammatory responses and neuronal protective machinery following TBIinduced ischemia in multiple types of mammalian cells, such as glial (Chen et al., 2011; Ouyang 
et al., 2013; Walker and Tesco, 2013). Additionally, miR-34a has been demonstrated to regulate glial via neural stem cell differentiation (Arnha et al., 2011). Furthermore, miR-27b serves as a critical regulator of mitochondrial function (Tak et al., 2014; Zhou et al., 2015). In addition, miR-34a exhibited key functions in regulation the keratinocyte layer of epithelium (Lefort et al., 2013). As such, we explore the possibility for these six miRNAs severing as mTBI biomarkers from hair follicles.

Using an Advanced Blast Simulator (ABS) system, we were able to induce primary blastinduced mTBI in a rat model by head-only exposure to single-pulse shockwaves at a range of pressure intensities (Zhang et al., 2014, 2018; Sawyer et al., 2016). Four blast intensities ranging from 15 to 30 psi were used to represent the shockwave exposure observed during a low-level far-field explosion event (Sawyer et al., 2016). Based on the publically available microarray gene expression profiles (NCBI Gene Expression Omnibus (GEO) database Accession ID: GSE80371) established from our previous study (Zhang et al., 2018), we utilized gene set analysis approaches such as Sub-network enrichment analysis (SNEA) and Gene set enrichment analysis (GSEA) to assess and functionally characterize the aforementioned miRNAs of interest. Therefore, this study leverages bioinformatics and the low throughput qPCR amplification to explore the miRNA responses in the mammalian hair follicles. As such, we present a comprehensive evaluation of the mTBI response of the selected key miRNAs. 


\section{Materials and methods}

A flowchart is used to present the materials, exposure procedure and analysis process (Fig. S1).

Animal experiment

Adult male Sprague-Dawley (SD) rats (Rattus norvegicus) (aged 9 - 10 weeks, weight 350 - 400 g) were obtained from Charles River Laboratories (St. Constant, Que, Canada) and used for the study. Rats were grouped into five per experimental conditions. A custom-built Advanced Blast Simulator (ABS) system (Defence Research and Development Canada, or DRDC, AB, Canada) was used for all single intensity shockwave exposures (Zhang et al., 2014; Sawyer et al., 2016). All animals were acclimated under a controlled laboratory environment for a minimum of one week before use. Rats from both control ( 0 psi) and experimental groups were treated with 3\% isoflurane anaesthetic (mixed with oxygen) for 8 min and stabilized in a plastic sleeve prior to the exposure. For shockwave experiments, sleeves were placed in the ABS chamber with the rat's head in the test area for an accurate head impact. Shockwave intensities at 0 psi (control), 15, 20, 25, and 30 psi were used for the exposure $(\mathrm{N}=5)$.

General health conditions and signs of stress were monitored in each animal following exposure and before sample collection at 24 hours post-exposure. Up to 20 whisker follicles per rat were collected and stored in RNA later solutions (Qiagen, ON, Canada) for all experimental groups. Animal experiments were conducted under the guidelines from ' 'Guide to the Care and Use of Experimental Animals” and “The Ethics of Animal Experimentation” by Canadian 
Council on Animal Care. All experimental protocols were approved by the Animal Care Committee at DRDC.

\section{Microarray analysis}

Whisker follicle RNA was isolated using the RNeasy Micro Kit (Qiagen, ON, Canada) for miRNA amplification. Briefly, whisker follicles were homogenized using a sonicator (ThermoFisher, ON, Canada) and the RNA was purified using HiBind RNA Spin Columns associated with the RNA extraction kits. A Nanodrop-2000 instrument (ThermoFisher, ON, Canada) was used to determine the concentration and purity of the RNA samples, while the integrity was examined with a Bioanalyzer instrument (Agilent, ON, Canada) and presented as RNA integrity numbers (RINs). RNA samples with a minimum RIN of 7 were used for further analyses.

The whisker follicle gene expression profiling data were collected, validated and submitted by our group previously (Zhang et al., 2018) (GEO Accession ID: GSE80371). Briefly, the microarray was conducted using the Agilent SurePrint G3 Rat GE 8 x 60 K system (Agilent, ON, Canada). Samples were prepared with 200 ng total RNA using the Agilent Low RNA Input Fluorescent Amplification Kit (Agilent, ON, Canada). Raw expression data were processed and analyzed using JMP Genomics 6.0 software (SAS, NC, USA). Gene level statistics (differential expression or DE analysis) were calculated using One-way analysis of variance (ANOVA) after Quantile normalization and removal of control data in JMP Genomics.

miRNA sub-network enrichment analysis (SNEA) 
Sub-networks for the miRNAs of interest were constructed using the knowledge-based SNEA function in Pathway Studio 10 (Elsevier, USA). Based on the gene expression profiles, SNEA utilizes the regulatory relationships extracted from most up-to-date literature database ResNet (Elsevier, USA) to enrich regulated pathways. This approach branches beyond the measured gene expression data, to explore the potential upstream regulators responsible for the observed gene level changes (Sivachenko et al., 2007). The literature-driven sub-network building proposes an unbiased miRNA-mRNA regulatory relation upon the head physical trauma exposure. Furthermore, the specific exposure conditions for which the miRNA level was measured and compared were also determined by SNEA. The "best $p$ value (the most significant change)" was set for SNEA in the case of duplicated probes in microarray. The alpha level of SNEA was set at 0.05 and an FDR correction was applied for the analysis. Additionally, visual representations of miRNA-subnetworks were generated with the expression levels masked for the target genes.

miRNA amplification with quantitative RT-PCR (qPCR)

qPCR was used to amplify and compare the six miRNAs revealed by SNEA in the rat whisker follicles in response to the shockwave exposures, with let-7a used as a reference gene. Mature sequences for rat miRNAs (only the dominant form) miR-183 (rno-miR-183-5p), miR26a (rno-miR26a-5p), miR-181c (rno-miR-181c-5p), miR-29a (rno-miR29a-3p), miR-34a (rnomiR34a-5p), miR-27b (rno-miR-27b-3p) as well as let-7a (rno-let-7a-5p) were obtained from miRBase (www.mirbase.org/) as the basis for primer design. Primer sets were designed for each miRNA as previously described by Balcells et al. (2011) with the melting temperature adjusted to $59{ }^{\circ} \mathrm{C}$. 
An RNA poly-adenylation step was added prior to cDNA synthesis using a Poly(A) Polymerase Tailing Kit (Epicentre Technologies, IL, USA). Each poly(A) tailing reaction contained $200 \mathrm{ng}$ total whisker follicle RNA and1 U poly(A) polymerase provided in the kit. The reactions were conducted at $37^{\circ} \mathrm{C}$ for $30 \mathrm{~min}$. Poly(A)-RNA samples were then subject to firststrand cDNA synthesis using the QuantiTect Reverse Transcription Kit (Qiagen, ON, Canada). A RT primer was used for cDNA synthesis for targeting poly-adenylated RNA.

A BioRad CFX thermocycler (BioRad, ON, Canada) was used for the qPCR, with the associated CFX manager software for signal quantification. All qPCR reactions were carried out using the GoTaq qPCR master mix (Promega, WI, USA), with a cycling condition of $95^{\circ} \mathrm{C}$ for $10 \mathrm{~min}$, followed by 40 cycles of $95^{\circ} \mathrm{C}$ for $30 \mathrm{sec}$ and $60^{\circ} \mathrm{C}$ for $1 \mathrm{~min}$. A dissociation curve was generated over the temperature range of $60-95^{\circ} \mathrm{C}$ immediately after the qPCR reaction to ensure single product amplification. All the sequences and the optimized qPCR condition for each miRNA are provided in Table 1. Amplification of the miRNA targets of interest was verified via cloning. Table 1 also shows the major biological functions for the miRNAs tested.

One-way ANOVA with Dunnett's test was used for statistical analysis for the qPCR amplification using the JMP SAS 9.0 software (SAS, NC, USA). Changes with a $p$ value less than 0.05 were considered statistically significant.

Gene set enrichment analysis (GSEA)

GSEA was used to assess the functional significance of the miRNAs tested via the genes identified by the miRNA sub-networks. As proposed originally by Subramanian et al. (2005, software.broadinstitute.org/gsea/), GSEA utilizes pre-defined gene sets to correlate the individual 
gene expression data to the specific pathways and biological processes. The analysis was conducted on the genes that were enriched in the SNEA-identified miRNA sub-networks. Specifically, gene ontology term (GO) gene sets were used with biological processes (BP) and molecular function (MF) terms assessed separately. To thoroughly characterize the functional impact from miRNA-related gene expression regulation, additional gene set collections featured in ResNet were queried with GSEA, including cell process, receptor pathways, cell signaling, immune pathway, metabolic pathway, and nociception pathway. The GSEA gene set significance was determined using a Kolmogorov-Smirnov classic permutation approach (1000 times), with rat genome as the background list. Similar to SNEA, the lowest gene level $p$ value was used for duplicated probes in microarray. Pathways with a FDR corrected $p$ value less than 0.05 was considered significantly enriched.

\section{Results}

\section{Animal Exposures}

All rats were exposed to mTBI-inducing blast regime as described in Zhang et al. (2014, 2018) and Sawyer et al. (2016). Animals exposed to simulated blast (15, 20, 25, 30 psi) showed little or no effects of treatment compared to sham controls with respect to time-to-awake, timeto-mobile and time-to-normal (grooming, exploratory behaviour) values. No significant changes in weight were noted in any treatment group compared to sham controls.

Sub-network enrichment analysis (SNEA) 
SNEA was used to construct miRNA-dependent regulatory sub-networks for miR-183, miR-26a, miR-181c, miR-29a, miR-34a, and miR-27b based on the microarray data. The test was carried out using the gene level statistics ( $p$ values and fold change) generated from a rat $8 \mathrm{x}$ $60 \mathrm{~K}$ gene chip over four shockwave intensities (i.e., 15, 20, 25, and 30 psi), all relative to the 0 psi control levels. Indeed, all six miRNAs were significantly enriched by SNEA with their respective downstream genes and exposure conditions (Table 2). It is worth noting that the enriched sub-networks for all six miRNAs showed a high mRNA target coverage, with more than $70 \%$ of total downstream mRNA targets measured by microarray. All six miRNA-related sub-networks can be viewed in Figs. 1-4. The complete SNEA results can be found in Appendix 1. SNEA also revealed additional miRNA sub-networks that were hypothesized to be related to the transcriptomic responses observed in microarray either universal or specific to the exposure intensity, a summary of which can be viewed in Fig. S2.

miRNA qPCR amplification

To explore the potential molecular mechanism behind the sub-network enrichment, the transcript level of the six core miRNAs were measured in the hair follicles under the corresponding shockwave exposure conditions using qPCR. Since the miR-183 sub-network was enriched in all exposure conditions, the hair follicle transcript levels of miR-183 from all four experimental groups were compared with the control level (Fig. 5A). When comparing with the 0 psi control group, the level of miR-183 significantly decreased to $68.7 \pm 7.1 \%$ of the control level under the 15 psi exposure ( $p<0.05$; Fig. 5A). Relative miR-183 levels exhibited a dramatic reduction in the 20 psi condition to $8.9 \pm 2.5 \%$ of the control value $(p<0.05)$, followed by an increase to $66.4 \pm 1.4 \%$ in the 25 psi group relative to the control group. In the 30 psi group, 
miR-183 transcript levels also showed a significant decrease of $36.3 \pm 1.1 \%$ of the 0 psi control level. As for the miR-26a sub-network enriched in hair follicle from the rats exposed to 15, 20, and 30 psi shockwaves, it appears that only the 30 psi condition led to a significant reduction in the miR-26a levels to $54.3 \pm 5.2 \%$ of the control level $(p<0.05)$ (Fig. 5B). Fig. 5C is a representative histogram for the relative transcript levels of miR-181c in the hair follicles exposed to 15 psi and 25 psi shockwaves. A significant reduction in miR-181c levels to $66.5 \pm$ 3.6\% was observed in the hair follicles from the 15 psi shockwave exposed the group $(p<0.05)$. The lowest miR-181c levels were observed in the 25 psi exposed hair follicles, at only $18 \pm 4.2 \%$ of the control level $(p<0.05)$.

Fig. 6 depicts the relative expression levels of the miRNAs of interest (i.e., miR-29a, miR-34a, and miR-27b) whose sub-network was only enriched under one specific exposure condition by SNEA. As seen in Fig. 6A, the relative miR29a levels were significantly reduced to $26.6 \pm 3.2 \%$ of the control group upon 15 psi shockwave exposure $(p<0.05)$. A significant decrease of the miR-34a level was also observed in the hair follicles from the 20 psi exposed group, and these were reduced to $58.9 \pm 2.2 \%$ of the values in the control group $(p<0.05)$ (Fig. 6B).

\section{Gene set enrichment analysis (GSEA)}

The mRNA targets presented in the six miRNA sub-networks were subject to GSEA with their expression levels, by which the biological and functional significance of the miRNA subnetworks was assessed. Multiple gene set databases were tested for a comprehensive survey regarding to the biological significance of the miRNA-dependent regulatory sub-networks. Specifically, GO terms (BP and MF), cell process, receptor pathways, cell signaling, immune 
pathway, metabolic pathway, and nociception pathway were explored. Table 3 shows selected pathways that are associated to molecular signatures for potential mTBI-associated neurodegeneration.

For the miR-183 sub-networks that were proposed in all four exposure conditions, GSEA on the measured gene targets revealed a number of gene sets from the aforementioned collections (Table 3 and Appendix 2A). Specifically, the 15 psi shockwave exposure led to enrichment of the GO terms that included small GTPase mediated signal transduction (BP, GO: 0007264), cell cycle (BP, GO: 0007049), voltage-gated potassium channel activity (MF, GO: 0005249), and voltage-gated calcium channel activity (MF, GO: 0005245). In the 20 psi exposed hair follicles, the enriched GO terms included negative regulation of cellular calcium ion homeostasis (BP, GO: 0006874), apoptotic signaling pathway (BP, GO: 0097190), central nervous system neuron axonogenesis (BP, GO: 0021955), and synaptic transmission (BP, GO: 0007268). For the 25 psi exposure, GO term analysis using GSEA showed enrichment of cell death (BP, GO: 0008219), cellular response to cAMP (BP, GO: 0071320), and potassium ion transmembrane transport (BP, GO: 0071805), as well as MF terms including protein kinase activity (BP, GO: 0004672), and transcription factor binding (BP, GO: 0008134). Additionally, the GSEA results on the cell signaling set collection also suggested enriched pathway like insulin action under 25 psi exposure. Moreover, 30 psi led to GO term enrichment of DNA repair (BP, GO: 0006281), ion transport (BP, GO: 0006811), neuron migration (BP, GO: 0001764), protein kinase binding (MF, GO: 0019901), and receptor binding (MF, GO: 0005102).

The GSEA test was conducted on the measured targets in the miR-26a sub-network, which was enriched under 15, 20, and 30 psi exposures (Table 3 and Appendix 2B). For the miR26 sub-network enriched in the 15 psi shockwave exposed hair follicles, the over-represented GO 
terms included cell cycle (BP, GO: 0007049), keratinocyte differentiation (BP, GO: 0030216), receptor binding (MF, GO: 0005102), and metal ion binding (MF, GO: 0046872). It is worth noting that $\mathrm{T}$-cell receptor signaling featured in the immunological pathway collection was also enriched for the miR-26 sub-network under 15 psi exposure. For the 20 psi exposed animals, the miR-26a sub-network exhibited enrichment of glutamate receptor binding (BP, GO: 0035254), hair cycle (BP, GO: 0022405), negative regulation of glial cell apoptotic process (MF, GO: 0034351), and glutamate receptor binding (MF, GO: 0035254). The 30 psi exposed group saw the enrichment of the insulin receptor signaling pathway (BP, GO: 0008286), cellular response to ATP (BP, GO: 0071318), ATP binding (MF, GO: 0005524), mitochondrial outer membrane permeabilization (BP, GO: 0097345), and nucleotide binding (GO: 0000166). Moreover, epidermal growth factor receptor (EGFR)/mothers against decapentaplegic homolog 1 (SMAD1) signaling was also enriched.

Table 3 and Appendix 2C contain the GSEA results for miR-181c sub-networks enriched in the hair follicle samples. As suggested by SNEA, both 15 and 25 psi groups were tested specifically. Upon 15 psi exposure, GO terms positive regulation of G-protein coupled receptor protein signaling pathway (BP, GO: 0045745), positive regulation of MAP kinase activity (BP, GO: 0043406), and cytosolic calcium ion homeostasis (BP, GO: 0051480) were significantly enriched. In the case of 25 psi exposure, GO terms RNA export from nucleus (BP, GO: 0006405), glutamate receptor activity (BP, GO: 0008066), G-protein coupled glutamate receptor signaling pathway (BP, GO: 0007216), regulation of long-term neuronal synaptic plasticity (BP, GO: 0048169), and activation of MAPKKK activity (BP, GO: 0000185) were enriched.

The measured mRNA targets (from microarray data) in the shockwave intensity-specific miRNA sub-networks were also assessed using GSEA (Table 3 and Appendix 2D - F). The 
measured downstream targets of the 15 psi-specific miR-29a sub-network saw the enrichment of GO terms involved in the RNA-dependent gene expression regulation such as nucleartranscribed mRNA catabolic process, transcription from RNA polymerase II promoter (BP, GO: 0006366), and RNA polymerase II transcription factor binding (MF, GO: 0001085). The results also showed enrichment of GO terms related to neuronal responses, including neuron-neuron synaptic transmission (BP, GO: 0007270), negative regulation of neurogenesis (BP, GO: 0050768), and long term synaptic depression (BP, GO: 0060292). Additionally, ionic responsesrelated GO terms were also enriched, including detection of calcium ion (BP, GO: 0005513), positive regulation of sodium ion transport (BP, GO: 0010765), and calcium channel regulator activity (MF, GO: 0005246). Moreover, GSEA on receptor pathway enriched NTRK (Neurotrophic Tyrosine Receptor Kinase)/ FOXO (Forkhead box O)/MYCN (N-myc protooncogene protein) signaling. The $20 \mathrm{psi}$ - specific miR-34a sub-network enriched GO terms including hair follicle morphogenesis (BP, GO: 0031069), keratinocyte differentiation (BP, GO: 0030216), regulation of synaptic plasticity (BP, GO: 0048167), glial cell apoptotic process (BP, GO: 0034349) and positive regulation of release of cytochrome c from mitochondria (BP, GO: 0090200) (Table 3 and Appendix 2E). Regarding to the mRNA targets in the 25 psi - specific miR-27b sub-network, GSEA enrichment led to over-representation of the GO terms protein folding (BP, GO: 0006457), and activation of signaling protein activity involved in ionotropic glutamate receptor signaling pathway (BP, GO: 0035235), regulation of synaptic plasticity (BP, GO: 0048167), calcium ion transport (BP, GO: 0006816), glutamate receptor binding (MF, GO: 0035254), and tau-protein kinase activity (MF, GO: 0050321).

\section{Discussion}


Mammalian hair follicles respond to primary blast-derived mTBI on a transcriptome level and genes related to ionic flux, cellular stress signal transduction, cell proliferation, apoptosis, immune responses, and regulation of the central nervous system are differentially expressed (Zhang et al., 2014, 2017). Due to their ubiquitous expression patterns and stability, miRNAs have been explored as potential biomarkers and therapeutic targets for diseases (Mitchell et al., 2008; Wang, 2009; Adachi et al., 2010; Hanke et al., 2010). We combined both the targetspecific (i.e. qPCR) and high-throughput (i.e. comprehensive bioinformatics analysis on microarray gene expression profiling) approaches to assess and characterize miRNA response in rat whisker hair follicles upon primary blast induced-mild traumatic brain injury.

All six miRNAs of interest were enriched as miRNA-mRNA regulation networks by SNEA. The miR-183, miR-26a, and miR-181c sub-networks were enriched in multiple shockwave intensities, with the rest specific to one. Additionally, miR-27b, miR-29a, miR-34a, and miR-183 contained a sizeable number of downstream genes (over 100) according to ResNet database. Since the database is knowledge-based, a large number of downstream mRNA targets generally translate into a diverse number of pathways and cellular events subject to the regulations from the miRNAs of interest.

For the universally enriched miR-183 sub-network, GSEA suggested that its sub-network was involved in established mTBI responsive pathways and cellular processes under the exposure conditions tested. For example, the 15 psi exposure resulted in the enrichment of GO terms that included GTPase mediated signal transduction and cell cycle, both of which have been proposed to be mTBI responsive (Zhang et al., 2014). Importantly, the over-representation of the GO terms linked to cellular calcium and potassium transport across all exposure conditions suggests that the hair follicle miR-183 sub-network might be able to reflect blast-derived calcium 
overload and potassium-dependent neuroprotection mechanisms against mTBI-induced membrane depolarization. In addition, the sub-network analysis also exhibited enrichment of the GO terms connecting to apoptosis and axonogenesis, indicating that miR-183 sub-network might not only be reflective of the neuronal cell loss following mTBI (Giza and Hovda, 2014), but also participate in the coping mechanisms. Furthermore, decreased miR-183 levels were observed for all the intensities tested. The general trend revealed that the miR-183 transcript level decreased consistently with the increase of the shockwave intensity, with the exception of the $20 \mathrm{psi}$ condition, suggesting that this miRNA may be a master regulator of transcriptome responses following shock wave blasts.

For miR-26a, mRNA targets associated with the sub-network enriched GO terms linked to hair follicle/epidermis-specific keratinocyte differentiation in the15 psi group according to GSEA, consistent with the previous finding in which miR-26a was proposed as part of the keratinocyte regulation machinery (Hildebrand et al, 2011). In terms of both $20 \mathrm{psi}$ and $30 \mathrm{psi}$ groups, the miR-26a sub-network exhibited over-presented GO terms themed cellular calcium homeostasis and glial apoptosis. With the enrichment of the cellular events of glutamate receptor regulation and synaptic transmission, it appeared that miR-26a sub-network might be responsive to mTBI-induced glutamate excitotoxicity due to calcium imbalance, which ultimately leads to neurotransmission impairment (Walker and Tesco, 2013). However, no appreciable changes were observed in the transcript levels of the hair follicle miR-26a in both15 and 20 psi exposed rats when compared to the control group. Such results indicate that the enrichment of miR-26a sub-networks under 15 and 20 psi may occur by mechanisms other than the regulation of miRNA abundance, such as the adaptation of the interaction with the RNA binding proteins (Ciafre and Galardi, 2013). Under 30 psi exposure, however, the miR-26a transcript level was reduced to 
approximately $50 \%$ of the control level, suggesting that the regulation of miR-26 might be required to reach a high downstream gene coverage percentage under more severe traumatic conditions. Given the profound involvement of miR-26a in facilitating cell stress survival (Xi, et al., 2006; Kota et al., 2009; Lezina et al., 2013), the results agreed with our previous observation in which 30 psi exposure triggered the most cellular damage repair pathways and related cellular events in hair follicles (Zhang et al., 2018).

Besides the pathways linked to TBI responsive cellular events proposed previously (Zhang et al., 2014, 2018), GSEA on the miR-181c sub-network also featured chronic inflammatory response- and neuroinflammation-related GO terms in both 15 and 25 psi group, agreeing with a previous study (Zhang et al., 2012). Similar to miR-183 and miR-26a, calcium imbalance is another major theme enriched for miR-181c sub-networks in both exposure conditions. Indeed, this process is part of the molecular pathology of mTBI (Giza and Hovda, 2014). Additionally, for the 25 psi exposure group, glutamate receptor responses and neuronal synaptic plasticity were also enriched. As subcellular injury starts from calcium overload and glutamate receptor dysfunction, eventually leading to altered synaptic plasticity (Giza and Hovda, 2014), alterations in the miR-181c sub-network may be reflective to mTBI progression. Furthermore, miR-181c transcript level showed a consistent and significant reduction as the shockwave increased from 15 to 25 psi, when comparing with the control animals. As such, the results exhibited a connection between the miRNA transcript level and stress severity. Therefore, the hair follicle miR-181c appeared to be reflective of the exposure conditions.

Regarding the 15 psi exposure-specific miR-29a sub-network, GSEA revealed a striking 327 over-represented GO BP terms and 87 MF terms. Specifically, enriched pathways included transcriptional regulation of RNA, and RNA-dependent transcription regulation pathways and 
cell proliferation, both of which were proposed previously for the hair follicles from all four exposure conditions (Zhang et al., 2018). It is worth noting that several FOXO related receptor signaling (e.g., NTRK/FOXO/MYCN signaling) were also enriched, indicating that the mTBI responsive FOXO signaling might be part of the miR-29a sub-network function. In addition, the miR-29a sub-network also provided signs of mTBI-derived cellular ionic responses, synaptic transmission, and inflammatory responses. Moreover, mitochondria-induced apoptosis responses were identified upon 15 psi exposure. Furthermore, the enrichment of neuroblast proliferation implies the involvement of miR-29a sub-network in the cell cycle-dependent neuro-protective mechanisms against neuronal cell loss following mTBI. In addition, the transcription level of the hair follicle miR-29a dropped significantly comparing to the control level. Due to the posttranscriptional inhibitory effect of miRNAs on their downstream mRNAs, the decrease of the miR-29a transcript level was consistent with the SNEA results where more increased targets were present in the sub-network.

With the enrichment of the cell process adherens junction assembly (cadherins), the miR34a sub-network may be involved in the previously proposed 20 psi-specific BBB-related mTBI responses (Schulze and Firth, 1993; Chodobski et al., 2011; Abdul-Muneer et al., 2013; Zhang et al., 2016, 2018). It is also worth noting that the miR-34a sub-network enriched multiple hair cycle response-related GO terms. Given the roles of miR-34a in regulating keratinocyte function (Lefort et al., 2013), our results suggest that this miRNA reflects the potential mTBI impact to hair follicle/epidermis at 20 psi. Furthermore, GO terms linked to glial apoptosis and synaptic transmission were also enriched, exhibiting signs of potential mTBI-associated neurodegenerative effect at 20 psi exposure. The GSEA results also revealed potential neuroprotective functions of the miR-34a sub-network through the enrichment of the GO term 
axonogenesis. Similar to miR-29a, a significant decrease in the transcript level of miR-34a was suggested by qPCR. Such decrease of miR-34a level was accompanied by the more increased downstream target levels and might be part of the molecular mechanism behind the 20 psi enrichment by SNEA.

For the 25 psi enriched miR-27b sub-network, over-presentation of the GO terms such as calcium transport, glutamate receptor responses and mitochondrial apoptosis pathways were observed (Zhang et al., 2018). These indicate the sub-network being able to represent the overall mTBI progression at 25 psi exposure. Unique to 25 psi intensity and the miR-27b sub-network, the theme of the tau protein response was enriched through the GO term tau-protein kinase activity. It has been established that hyper-phosphorylation of tau is a shared molecular signature between mTBI and Alzheimer's disease (AD) (Torres-Aleman, 2008; Johnson et al., 2012). With the involvement of miR-27b in the pathology of neurodegenerative diseases (Maciotta et al., 2013), our observation suggested that the miR-27b sub-network might be able to reflect such signatures in the primary blast-induced mTBI at 25 psi. The qPCR result for miR-27b, however, suggested a distinctive regulation pattern in the 25 psi shockwave exposed hair follicles. Despite of the significant miR-27b sub-network enrichment, the transcript level of miR-27b maintained comparable to the control level. However, over $80 \%$ measured gene coverage with a total gene count of 102 still suggested a considerable mTBI response of the current sub-network (Table 2). The discrepancy between SNEA and qPCR results indicated that other mechanisms beyond transcriptional control on miR-27b might be responsible for the enrichment of the sub-network.

\section{Conclusions}


As previously established mTBI molecular signatures, the rat whisker hair follicle miR183, miR-26a, miR-181c, miR-29a, miR-34a, and miR-27b also seemed to be responsive to the mTBI-inducing single-pulse shockwave head exposures. Firstly, the SNEA results not only confirmed that all six miRNAs participate in regulating TBI-responsive transcriptomic adaptations, but also identified the corresponding overpressure intensities associated with the enriched miRNA sub-networks. Secondly, our GSEA results suggested that enriched miRNA ubnetworks were reflective of the signature cellular responses to mTBI. The present study also revealed that on miR-181c (15 psi), miR-26a (20 psi), miR-181c (25 psi), and miR-27b (25 psi) centered sub-networks might be capable of exhibiting mTBI progression. Accordingly, the present study demonstrated for the first time that mammalian hair follicle was capable of providing potential miRNA signatures for primary blast-derived mTBI in an overpressure intensity-sensitive fashion.

\section{Clinical significance}

First the first time, the current study provided evidence to using hair follicles as a miRNA biomarker discovery tool for mTBI. As such, a valuable direction for future studies is to assess the viability of the hair follicle miRNAs investigated in the current study as a diagnostic tool for mTBI patients.

\section{Funding details}


This research was supported by the Defence Research and Development Canada (DRDC) Technology Investment Fund (TIF) to VSL (PI), CJM and YW. VSL holds a Canadian Research Chair.

\section{Acknowledgements}

The authors thank the excellent technical assistance by Tracy Weiss, Peggy Nelson, Grant Hennes, Cory Vair and Julia Barnes at DRDC during animal exposure to blast, as well as Sarah Wallace (RMCC) for molecular biology technical assistance.

\section{Disclosure Statement}

The authors report no conflict of interest 


\section{References}

Abdul-Muneer PM, Schuetz H, Wang F, et al. (2013) Induction of oxidative and nitrosative damage leads to cerebrovascular inflammation in an animal model of mild traumatic brain injury induced by primary blast. Free Radic Biol Med. 60: 282 - 291.

Adachi T, Nakanishi M, Otsuka Y, et al. (2010) Plasma microRNA 499 as a biomarker of acute myocardial infarction. Clin Chem 56(7):1183 - 1185.

Ahmed MI, Alam M, Emelianov VU, et al. (2014) MicroRNA-214 controls skin and hair follicle development by modulating the activity of the Wnt pathway. J Cell Biol 207(4): 549 - 67.

Aranha MM, Santos DM, Solá S, et al. (2011) miR-34a regulates mouse neural stem cell differentiation. PLoS One 6(8): e21396.

Balakathiresan N, Bhomia M, Chandran R, et al. (2012) MicroRNA let-7i is a promising serum biomarker for blast-induced traumatic brain injury. J Neurotrauma 29(7):1379 - 1387.

Balcells I, Cirera S, Busk PK (2011) Specific and sensitive quantitative RT-PCR of miRNAs with DNA primers. BMC Biotechnol 11: 70.

Bramlett HM, Dietrich WD (2004) Pathophysiology of cerebral ischemia and brain trauma: similarities and differences. J Cereb Blood Flow Metab 24: 133 - 150.

Chen K, Rajewsky N (2007) The evolution of gene regulation by transcription factors and microRNAs. Nat Rev Genet 8(2): 93 - 103.

Chen T, Li Z, Tu J, et al. (2011) MicroRNA-29a regulates pro-inflammatory cytokine secretion and scavenger receptor expression by targeting LPL in oxLDL-stimulated dendritic cells. FEBS Lett 585: $657-663$. 
Chodobski A, Zink BJ, Szmydynger-Chodobska J (2011) Blood-brain barrier pathophysiology in traumatic brain injury. Transl Stroke Res 2(4):492 - 516.

Ciafrè SA, Galardi S (2013) microRNAs and RNA-binding proteins: A complex network of interactions and reciprocal regulations in cancer. RNA Biol 10(6): 935 - 942.

Das S, Bedja D, Campbell N, et al. (2014) miR-181c regulates the mitochondrial genome, bioenergetics, and propensity for heart failure in vivo. PLoS One. 9(5):e96820.

Defense and Veterans Brain Injury Center (DVBIC) (2013) DoD worldwide numbers for TBI (non-combat and combat injuries). Available: http://www. dvbic.org/dod-worldwide-numbers-tbi

Friedman RC, Farh KK, Burge CB, et al. (2009) Most mammalian mRNAs are conserved targets of microRNAs. Genome Res 19: $92-105$.

Gao W, Sun W, Yin J, et al. (2017) Screening candidate microRNAs (miRNAs) in different lambskin hair follicles in Hu sheep. PLoS One 12(5): e0176532.

Gibbings DJ, Ciaudo C, Erhardt M, et al. (2009) Multivesicular bodies associate with components of miRNA effector complexes and modulate miRNA activity. Nat Cell Biol 11: $1143-1149$.

Giza CC, Hovda DA (2014) The new neurometabolic cascade of concussion. Neurosurgery Suppl 4: S24 - 33.

Gu QH, Yu D, Hu Z, et al. (2015) miR-26a and miR-384-5p are required for LTP maintenance and spine enlargement. Nat Commun 6: 6789.

Ha M, Kim VN (2014) Regulation of microRNA biogenesis. Nat Rev Mol Cell Biol 15(8): 509 524. 
Hanke M, Hoefig K, Merz H, et al. (2009) A robust methodology to study urine microRNA as tumor marker: microRNA-126 and microRNA-182 are related to urinary bladder cancer. Urol Oncol 28(6): $655-661$.

He L, Hannon GJ (2004) MicroRNAs: small RNAs with a big role in gene regulation. Nat Rev Genet 5(7): $522-531$.

Hildebrand J, Rütze M, Walz N, et al. (2011) A comprehensive analysis of microRNA expression during human keratinocyte differentiation in vitro and in vivo. J Invest Dermatol 131: $20-29$.

Hochfeld LM, Anhalt T, Reinbold CS, et al. (2017) Expression profiling and bioinformatic analyses suggest new target genes and pathways for human hair follicle related microRNAs. BMC Dermatol 17(1): 3.

Johnson VE, Stewart W, Smith DH (2012) Widespread $\tau$ and amyloid- $\beta$ pathology many years after a single traumatic brain injury in humans. Brain Pathol 22(2):142 - 149.

Kota J, Chivukula RR, O'Donnell KA, et al. (2009) Therapeutic microRNA delivery suppresses tumorigenesis in a murine liver cancer model. Cell 137(6): 1005 - 1017.

Krol J, Busskamp V, Markiewicz I, et al. (2010) Characterizing light-regulated retinal microRNAs reveals rapid turnover as a common property of neuronal microRNAs. Cell 141: 618 $-631$.

Laurer HL, McIntosh TK (1999) Experimental models of brain trauma. Curr Opin Neurol 12: $715-721$. 
Lee CT, Risom T, Strauss WM (2007) Evolutionary conservation of microRNA regulatory circuits: an examination of microRNA gene complexity and conserved microRNA-target interactions through metazoan phylogeny. DNA Cell Biol. 26(4): 209 - 218.

Lefort K, Brooks Y, Ostano P, et al. (2013) A miR-34a-SIRT6 axis in the squamous cell differentiation network. EMBO J 32(16):2248 - 2263.

Lezina L, Purmessur N, Antonov AV, et al. (2013) miR-16 and miR-26a target checkpoint kinases Wee1 and Chk1 in response to p53 activation by genotoxic stress. Cell Death Dis. 4: e953.

Li MW, Mruk DD, Cheng CY (2012) Gap junctions and blood-tissue barriers. Adv Exp Med Biol 763: $260-280$.

Ling G, Bandak F, Armonda R, et al. (2009) Explosive blast neurotrauma. J Neurotrauma 26(6): $815-825$.

Maciotta S, Meregalli M, Torrente Y (2013) The involvement of microRNAs in neurodegenerative diseases. Front Cell Neurosci7: 265.

Mitchell PS, Parkin RK, Kroh EM, et al. (2008) Circulating microRNAs as stable blood-based markers for cancer detection. Proc Natl Acad Sci USA 105: 10513 - 10518.

Nakagawa A, Manley GT, Gean AD, et al. (2011). Mechanisms of primary blast-induced traumatic brain injury: insights from shock-wave research. J Neurotrauma 28(6): 1101 - 1119. Ouyang YB, Xu L, Lu Y, et al. (2013) Astrocyte-enriched miR-29a targets PUMA and reduces neuronal vulnerability to forebrain ischemia. Glia 61(11): 1784 - 1794.

Perron MP, Provost P (2008) Protein interactions and complexes in human microRNA biogenesis and function. Front Biosci 13: 2537 - 2547. 
Rizos E, Siafakas N, Koumarianou A, et al. (2012) miR-183 as a molecular and protective biomarker for cancer in schizophrenic subjects. Oncol Rep 28: 2200 - 2204.

Sajja VSSS, Jablonska A, Haughey N, et al. (2018) Sphingolipids and microRNA Changes in Blood following Blast Traumatic Brain Injury: An Exploratory Study. J Neurotrauma. 35(2): 353 $-361$.

Sawyer T, Wang Y, Ritzel DV, et al. (2016) High fidelity simulation of primary blast: direct effects on the head. J Neurotrauma 33: 1181 - 1191.

Schulze C, Firth JA (1993) Immunohistochemical localization of adherens junction components in blood-brain barrier microvessels of the rat. J Cell Sci 104 (Pt 3): 773 - 782.

Sivachenko AY, Yuryev A, Daraselia N, et al. (2007) Molecular networks in microarray analysis. J Bioinform Comput Biol 5: 429 - 456.

Subramanian A, Tamayo P, Mootha VK, et al. (2005) Gene set enrichment analysis: a knowledge-based approach for interpreting genome-wide expression profiles. Proc Natl Acad Sci USA 102: 15545 - 15550 .

Tak H, Kim J, Jayabalan AK, et al. (2014) miR-27 regulates mitochondrial networks by directly targeting the mitochondrial fission factor. Exp Mol Med 46:e123.

Torres-Aleman I (2008) Mouse models of Alzheimer's dementia: current concepts and new trends. Endocrinol 149(12): 5952 - 5957.

Valadi H, Ekstrom K, Bossios A, et al. (2007) Exosome-mediated transfer of mRNAs and microRNAs is a novel mechanism of genetic exchange between cells. Nat Cell Biol 9: 654 - 659 . 
Valiyaveettil M, Alamneh YA, Miller SA, et al. (2013) Modulation of cholinergic pathways and inflammatory mediators in blast-induced traumatic brain injury. Chem Biol Interact 203(1): 371 $-375$.

Wang K, Zhang S, Marzolf B, et al. (2009) Circulating microRNAs, potential biomarkers for drug-induced liver injury. Proc Natl Acad Sci USA 106: 4402 - 4407.

Walker KR, Tesco G (2013) Molecular mechanisms of cognitive dysfunction following traumatic brain injury. Front Aging Neurosci 9;5:29.

Weickmann JL, Glitz DG (1982) Human ribonucleases. Quantitation of pancreatic-like enzymes in serum, urine, and organ preparations. J Biol Chem 257: 8705 - 8710.

Xi Y, Shalgi R, Fodstad O, et al. (2006) Differentially regulated micro-RNAs and actively translated messenger RNA transcripts by tumor suppressor p53 in colon cancer. Clin Cancer Res 12(7Pt1): $2014-2024$.

Zhang J, Carnduff L, Norman G, et al. (2014) Transcriptional profiling in rat hair follicles following simulated Blast insult: a new diagnostic tool for traumatic brain injury. PLoS One 9(8): e104518.

Zhang J, Knight R, Josey T, et al. (2018) Comprehensive assessment of shockwave intensity: Transcriptomic biomarker discovery for primary blast-induced mild traumatic brain injury using the mammalian hair follicle. Brain Injury 32(1): 123 - 134.

Zhang J, Wallace SJ, Shiu MY, et al. (2017) Human hair follicle transcriptome profiling: a minimally invasive tool to assess molecular adaptations upon low-volume, high-intensity interval training. Physiological Reports 5(23) pii: e13534. 
Zhang L, Dong LY, Li YJ, et al. (2012) The microRNA miR-181c controls microglia-mediated neuronal apoptosis by suppresing tumor necrosis factor. J Neuroinflammation 9: 211.

Zhang Y, Qin W, Zhang L, et al. (2015) MicroRNA-26a prevents endothelial cell apoptosis by directly targeting TRPC6 in the setting of atherosclerosis. Sci Rep 5: 9401.

Zhou X, Zuo S, Xin W (2015) miR-27b overexpression improves mitochondrial function in a Sirt1-dependent manner. J Physiol Biochem71:753 - 762. 


\section{Figure captions}

Figure 1. MiR-183 sub-network with the measured gene targets under corresponding exposure intensities. Red colour represents increase in the transcript level, whereas blue means decrease. Major organelles are displayed to show the sub-cellular distribution for the downstream mRNA targets. (A) 15 psi, (B) 20 psi, (C) 25 psi and (D)30 psi.

Figure 2. MiR-26a sub-network with the measured gene targets under corresponding exposure intensities. The rest information same as Fig.1. (A) 15 psi, (B) 20 psi and (C) 30 psi.

Figure 3. MiR-181c sub-network with the measured gene targets under corresponding exposure intensities. The rest information same as Fig.1. (A) 15 psi and (B) 25 psi.

Figure 4. MiR-29a (A), miR-34a (B) and miR-27 (C) sub-networks with the measured gene targets under corresponding exposure intensities. The rest information same as Fig.1.

Figure 5. Histograms showing relative miRNA levels for (A) miR-183, (B) miR-26a and (C) miR-181c under corresponding exposure conditions from qPCR amplification, i.e. control animal group vs exposed groups. Data are mean fold change $\pm \mathrm{SEM}$; changes with a $p$ value less than 0.5 are considered statistically significant.

Figure 6. Histograms showing relative miRNA levels for (A) miR-29a, (B) miR-34a and (C) miR-27b under corresponding exposure conditions from qPCR amplification, i.e. control animal group vs exposed groups. Data are mean fold change $\pm \mathrm{SEM}$; changes with a $p$ value less than 0.5 are considered statistically significant.

Figure S1. A flow chart showing the experimental design of the current study.

Figure S2. A Venn diagram depicting the quantity of both shared and shockwave exposurespecific SNEA enriched miRNA sub-networks. 
Table 1. Primer sequences for the miRNAs analyzed using qPCR with optimized conditions including annealing temperature and primer concentration.

\begin{tabular}{|c|c|c|c|c|}
\hline Gene & Sequence & $\begin{array}{l}\text { Annealing } \\
\text { Temperatu } \\
\text { re }\left({ }^{\circ} \mathrm{C}\right)\end{array}$ & $\begin{array}{l}\text { Primer } \\
\text { Concentratio } \\
\text { n (nM) }\end{array}$ & $\begin{array}{l}\text { Notable } \\
\text { biological } \\
\text { functions }\end{array}$ \\
\hline \multirow[t]{2}{*}{$m i R-183$} & Forward: 5’- ACAGTATGGCACTGGTAGA-3’' & \multirow{2}{*}{60} & 250 & \multirow{2}{*}{$\begin{array}{l}\text { Sensory organ } \\
\text { development }\end{array}$} \\
\hline & Reverse: 5'- TCCAGTTTTTTTTTTTTTTTAGTGAAT-3' & & 250 & \\
\hline \multirow[t]{2}{*}{$m i R-26 a$} & Forward: 5’- GCAGTTCAAGTAATCCAGGATA-3’' & \multirow[b]{2}{*}{60} & 250 & \multirow{2}{*}{$\begin{array}{l}\text { Keratinocyte } \\
\text { regulation } \\
\text { machinery }\end{array}$} \\
\hline & Reverse: 5’- GTCCAGTTTTTTTTTTTTTTTAGCC-3’ & & 250 & \\
\hline \multirow[t]{2}{*}{$\operatorname{miR}-181 c$} & Forward: 5'- GAACATTCAACCTGTCGGT -3' & \multirow[b]{2}{*}{60} & 250 & \multirow{2}{*}{$\begin{array}{l}\text { Neuro- } \\
\text { inflammation } \\
\text { regulation }\end{array}$} \\
\hline & Reverse: 5’- GGTCCAGTTTTTTTTTTTTTTTACTC -3’ & & 250 & \\
\hline \multirow[t]{2}{*}{$\operatorname{miR}-29 a$} & Forward: 5’- GCAGTAGCACCATCTGAA -3’' & \multirow{2}{*}{60} & 250 & \multirow{2}{*}{ Cell proliferation } \\
\hline & Reverse: 5’- CCAGTTTTTTTTTTTTTTTAACCGA-3' & & 250 & \\
\hline \multirow[t]{2}{*}{$\operatorname{miR}-34 a$} & Forward: 5’- GCAGTGGCAGTGTCTT -3’' & \multirow{2}{*}{60} & 250 & \multirow{2}{*}{$\begin{array}{l}\text { Brain Blood } \\
\text { Barrier regulation }\end{array}$} \\
\hline & Reverse: 5’- GTTTTTTTTTTTTTTTACAACCAGC-3’ & & 250 & \\
\hline \multirow[t]{2}{*}{$m i R-27 b$} & Forward: 5’- CAGTTCACAGTGGCTAAGT -3’' & \multirow[b]{2}{*}{60} & 250 & \multirow{2}{*}{$\begin{array}{l}\text { Involvement in } \\
\text { neurodegenerativ } \\
\text { e diseases }\end{array}$} \\
\hline & Reverse: 5'- CCAGTTTTTTTTTTTTTTTGCAGA -3' & & 250 & \\
\hline \multirow[t]{2}{*}{ let-7a } & Forward: 5’- AGCAGTGAGGTAGTAGGT-3' & \multirow[b]{2}{*}{60} & 250 & \multirow[b]{2}{*}{ Reference gene } \\
\hline & $\begin{array}{l}\text { Reverse: 5'- CCAGTTTTTTTTTTTTTTTAACTATACA - } \\
\text { 3' }\end{array}$ & & 250 & \\
\hline $\begin{array}{l}\text { RT } \\
\text { primer }\end{array}$ & 5’-CAGGTCCAGTTTTTTTTTTTTTTTVN-3’' & & & \\
\hline
\end{tabular}


Table 2. miRNA-mRNA regulation sub-network enriched by SNEA for miRNAs miR-183, miR-26a, miR-181c, miR-29a, miR-34a, and miR-27b. P-values are corrected using FDR based on the number of gene sets available on ResNet.

\begin{tabular}{lllllll}
\hline $\begin{array}{l}\text { Exposure } \\
\text { intensity (psi) }\end{array}$ & $\begin{array}{l}\text { Gene Set } \\
\text { Seed }\end{array}$ & $\begin{array}{l}\text { Total number of } \\
\text { mRNA targets }\end{array}$ & $\begin{array}{l}\text { Measured } \\
\text { mRNA targets }\end{array}$ & $\begin{array}{l}\text { Percentage } \\
\text { coverage }\end{array}$ & $\begin{array}{l}\text { Median } \\
\text { change }\end{array}$ & $\begin{array}{l}\text { Adjusted } \\
\text { p-value }\end{array}$ \\
\hline 15 & miR-26a & 49 & 41 & $83.7 \%$ & 1.018 & 0.044 \\
& miR-29a & 267 & 208 & $77.9 \%$ & 1.021 & 0.021 \\
& miR-183 & 134 & 106 & $79.1 \%$ & 1.069 & 0.026 \\
& miR-181c & 12 & 9 & $75.0 \%$ & 1.259 & 0.039 \\
& & & & & & \\
20 & miR-26a & 49 & 41 & $83.7 \%$ & 1.078 & 0.013 \\
& miR-34a & 192 & 163 & $84.9 \%$ & 1.057 & 0.032 \\
& miR-183 & 134 & 107 & $79.9 \%$ & 1.049 & 0.034 \\
& miR-181c & 12 & & & & \\
& miR-183 & 134 & 10 & $83.3 \%$ & 1.35 & 0.013 \\
& miR-27b & 102 & 104 & $77.6 \%$ & 1.044 & 0.015 \\
& & & 83 & $81.4 \%$ & 1.021 & 0.044 \\
& miR-183 & 134 & & & & 0.002 \\
& miR-26a & 49 & 104 & $77.6 \%$ & 1.074 & 0.032 \\
\hline
\end{tabular}


Table. 3. Enriched gene sets for the measured mRNA targets in the miRNA sub-networks under their corresponding exposure conditions by GSEA. P-values are corrected using FDR based on the number of gene sets available on ResNet.

\begin{tabular}{|c|c|c|c|c|}
\hline $\begin{array}{l}\text { miRNA sub- } \\
\text { network }\end{array}$ & $\begin{array}{c}\text { Intensity } \\
\text { (psi) }\end{array}$ & Name & Category & FDR Adjusted $p$-value \\
\hline \multirow{17}{*}{ miR-183 } & & voltage-gated calcium channel activity & MF & 0.01176 \\
\hline & 20 & synaptic transmission & $\mathrm{BP}$ & 0.00001 \\
\hline & & potassium ion transmembrane transport & $\mathrm{BP}$ & 0.00031 \\
\hline & & cellular calcium ion homeostasis & $\mathrm{BP}$ & 0.00148 \\
\hline & & axon extension & $\mathrm{BP}$ & 0.00346 \\
\hline & & potassium ion transport & $\mathrm{BP}$ & 0.00556 \\
\hline & & ion transport & $\mathrm{BP}$ & 0.00678 \\
\hline & & neuron migration & $\mathrm{BP}$ & 0.01932 \\
\hline & & axonogenesis & $\mathrm{BP}$ & 0.02581 \\
\hline & 25 & synaptic transmission & $\mathrm{BP}$ & 0.00001 \\
\hline & & central nervous system neuron axonogenesis & $\mathrm{BP}$ & 0.00041 \\
\hline & & potassium ion transmembrane transport & $\mathrm{BP}$ & 0.00046 \\
\hline & & cellular calcium ion homeostasis & $\mathrm{BP}$ & 0.00205 \\
\hline & & ion transport & $\mathrm{BP}$ & 0.00263 \\
\hline & & axon extension & $\mathrm{BP}$ & 0.00327 \\
\hline & & potassium ion transport & $\mathrm{BP}$ & 0.00331 \\
\hline & & voltage-gated ion channel activity & MF & 0.00001 \\
\hline
\end{tabular}




\begin{tabular}{|c|c|c|c|c|}
\hline & 30 & $\begin{array}{l}\text { synaptic transmission } \\
\text { central nervous system neuron axonogenesis } \\
\text { potassium ion transmembrane transport } \\
\text { cellular calcium ion homeostasis } \\
\text { ion transport } \\
\text { axon extension } \\
\text { potassium ion transport }\end{array}$ & $\begin{array}{l}\mathrm{BP} \\
\mathrm{BP} \\
\mathrm{BP} \\
\mathrm{BP} \\
\mathrm{BP} \\
\mathrm{BP} \\
\mathrm{BP}\end{array}$ & $\begin{array}{l}0.00001 \\
0.00041 \\
0.00046 \\
0.00205 \\
0.00263 \\
0.00327 \\
0.00331\end{array}$ \\
\hline miR-26a & 20 & $\begin{array}{l}\text { regulation of high voltage-gated calcium channel } \\
\text { activity } \\
\text { positive regulation of synaptic plasticity } \\
\text { mitochondrial outer membrane permeabilization } \\
\text { negative regulation of glial cell apoptotic process } \\
\text { negative regulation of calcium ion transport } \\
\text { negative regulation of axon extension } \\
\text { positive regulation of synaptic transmission, } \\
\text { glutamatergic } \\
\text { calcium ion homeostasis } \\
\text { glutamate receptor binding }\end{array}$ & $\begin{array}{l}\mathrm{BP} \\
\mathrm{BP} \\
\mathrm{BP} \\
\mathrm{BP} \\
\mathrm{BP} \\
\mathrm{BP} \\
\mathrm{BP} \\
\mathrm{BP} \\
\mathrm{MF}\end{array}$ & $\begin{array}{l}0.00698 \\
0.01045 \\
0.01218 \\
0.01218 \\
0.02423 \\
0.02423 \\
0.03781 \\
0.04286 \\
0.02200\end{array}$ \\
\hline & 30 & $\begin{array}{l}\text { regulation of high voltage-gated calcium channel } \\
\text { activity } \\
\text { negative regulation of synaptic transmission, } \\
\text { dopaminergic } \\
\text { positive regulation of synaptic plasticity } \\
\text { negative regulation of glial cell apoptotic process } \\
\text { mitochondrial outer membrane permeabilization } \\
\text { glutamate receptor binding } \\
\text { negative regulation of calcium ion transport } \\
\text { negative regulation of axon extension }\end{array}$ & $\begin{array}{l}\mathrm{BP} \\
\mathrm{BP} \\
\mathrm{BP} \\
\mathrm{BP} \\
\mathrm{BP} \\
\mathrm{BP} \\
\mathrm{BP} \\
\mathrm{BP}\end{array}$ & $\begin{array}{l}0.00758 \\
0.00758 \\
0.01134 \\
0.01218 \\
0.01322 \\
0.02398 \\
0.02628 \\
0.02628\end{array}$ \\
\hline
\end{tabular}




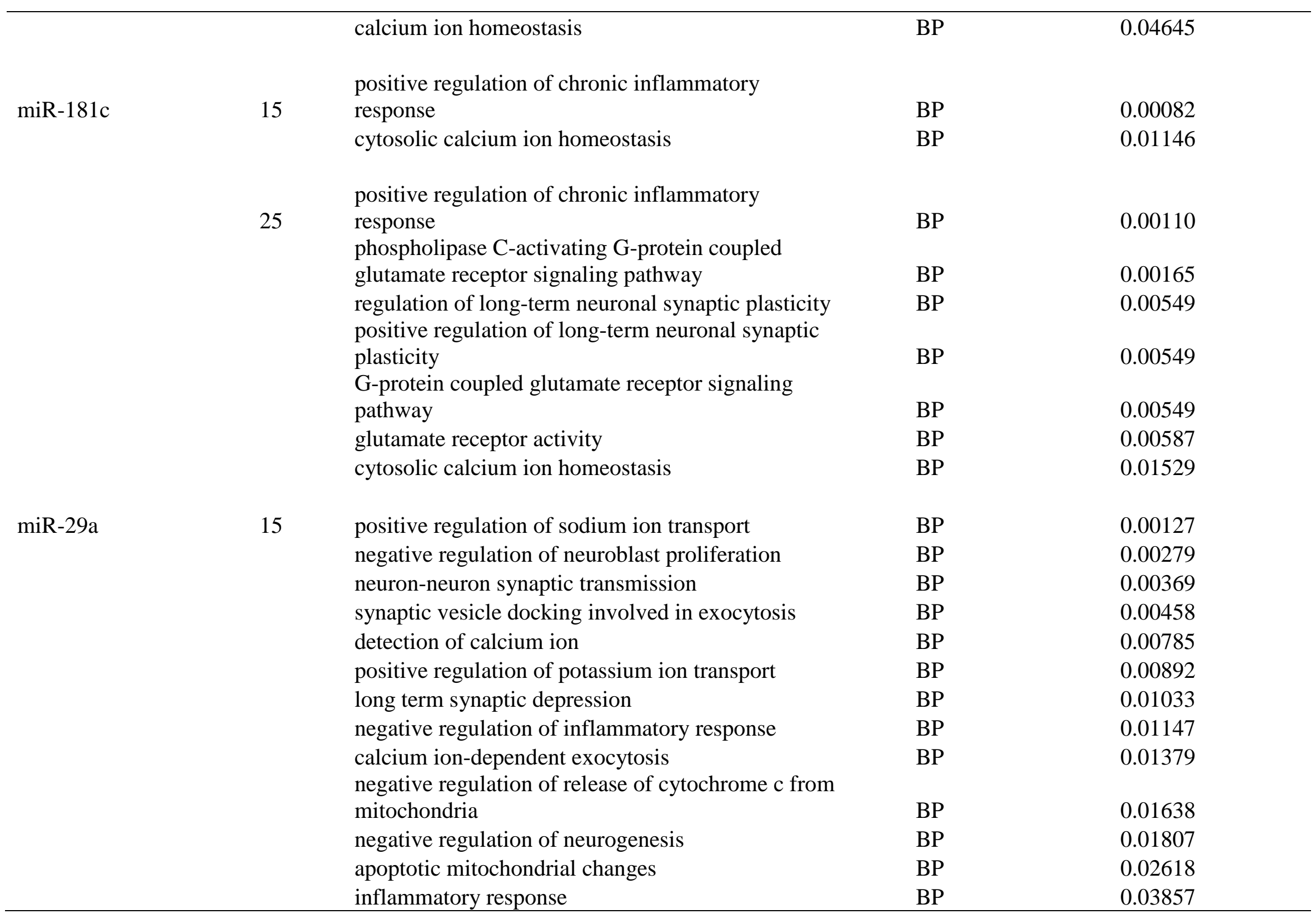




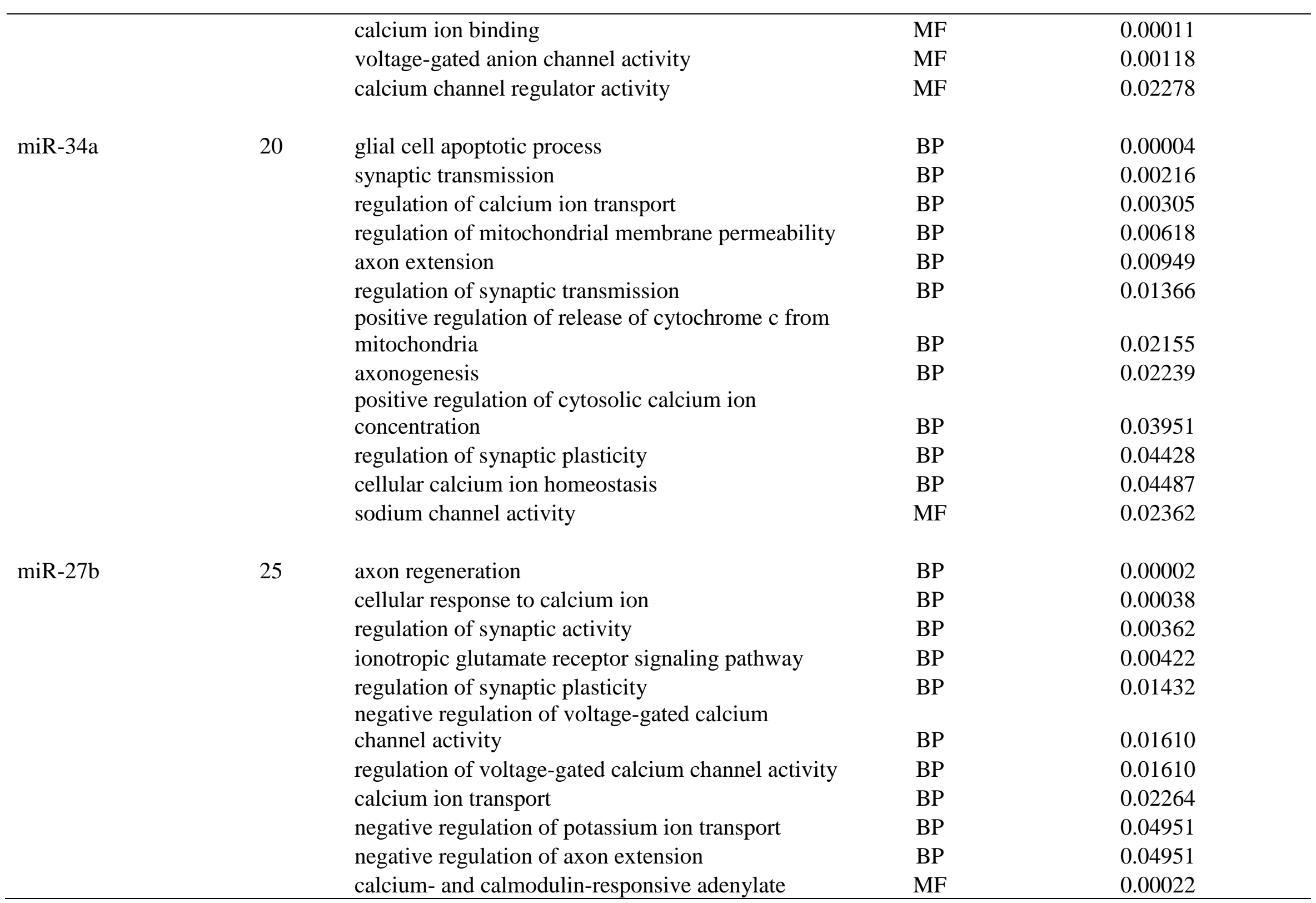


cyclase activity

type 3 metabotropic glutamate receptor binding

MF

0.01525

calcium-dependent protein binding

$\mathrm{MF}$

0.01939

tau-protein kinase activity

MF

0.04259

glutamate receptor binding

MF

0.04871

Mitochondrial Protein Transport

Cell Process

Pathways

0.00115 